\title{
Aging of Acute Deep Vein Thrombosis Measured by Radiolabeled ${ }^{99 m}$ Tc-rt-PA
}

\author{
Timothy Brighton ${ }^{1}$, Julia Janssen ${ }^{2}$, and S. Patrick Butler ${ }^{2}$ \\ ${ }^{1}$ Department of Haematology, St. George Hospital, Sydney, New South Wales, Australia; and ${ }^{2}$ Department of Nuclear Medicine, \\ St. George Hospital, Sydney, New South Wales, Australia
}

\begin{abstract}
In previous studies, ${ }^{99 m}$ Tc-recombinant tissue plasminogen activator (rt-PA) imaging has had a high sensitivity and specificity for the detection of deep vein thrombosis (DVT). In this technique, the plasminogen activation site of rt-PA undergoes inactivation but fibrin binding is retained. Uptake of $99 \mathrm{mTc}$-rt-PA into DVT relies on binding of $\mathrm{C}$-terminal lysine residues on fibrin. It is postulated that as the thrombus ages, fewer fibrin sites are available for ${ }^{99 \mathrm{~m}} \mathrm{Tc}$-rt-PA and that there should be a progressive decrease in 99mTc-rt-PA uptake in old thrombi as compared with fresh thrombi. The ability to differentiate fresh from old thrombus would have significant clinical implications in the objective diagnosis of recurrent DVT. Our aim was to examine the relative uptake of 99mTc-rt-PA in acute DVT over the first $30 \mathrm{~d}$ after diagnosis. Methods: Seventy-four patients with acute symptomatic DVT were entered into the study. Patients underwent ultrasound and ${ }^{99 m}$ Tc-rt-PA imaging on days 1, 7, and 30. Results: Residual thrombus was detected by ultrasonography in 46 (84\%) of 55 patients on day 7 and in 29 (66\%) of 44 patients on day 30. Of the persisting thrombi on day $7,72 \%(33 / 46)$ showed $99 \mathrm{mT}$ Tc-rt-PA uptake. Of the persisting thrombi on day $30,0 \%(0 / 29)$ showed 99mTc-rt-PA uptake. Conclusion: Uptake of $99 \mathrm{mTc}-\mathrm{rt}-\mathrm{PA}$ into DVT was absent $30 \mathrm{~d}$ after diagnosis. This finding suggests that this imaging technique can distinguish fresh from old thrombus.
\end{abstract}

Key Words: hematology; respiratory; vascular; imaging; recombinant tissue plasminogen activator; recurrent DVT

J Nucl Med 2007; 48:873-878

DOI: 10.2967/jnumed.106.039396

\section{D}

eep vein thrombosis (DVT) is a common condition afflicting 1 person per 1,000 every year $(1,2)$. DVT results in significant morbidity and mortality in the first $12 \mathrm{mo}$ related to pulmonary embolism, the emergence of underlying conditions such as malignancy, and complications from the anticoagulant therapy (3). The longer-term consequences of DVT are also a significant burden to the patient and the community at large. Almost one third of patients with a first DVT will develop recurrent DVT over an 8- to

Received Dec. 29, 2006; revision accepted Mar. 16, 2007.

For correspondence contact: S. Patrick Butler, MD, Department of Nuclear Medicine, St. George Hospital, Sydney, New South Wales, Australia, 2217. E-mail: sp.butler@unsw.edu.au

COPYRIGHT ( 2007 by the Society of Nuclear Medicine, Inc. 10-y period despite adequate initial anticoagulant therapy (3-6). Additionally, postthrombotic symptoms such as swelling, ache, immobility, stasis eczema, and venous ulceration are relatively common and are moderate or severe in $10 \%-$ $20 \%$ of patients $(7,8)$.

Age determination of DVT relies on the historical recall of symptoms. Unfortunately, patient recollection may be unreliable, and whereas ultrasound elasticity may allow differentiation between fresh and organized thrombus, such criteria have not been well validated $(9,10)$. Once formed, DVT undergoes several physiologic changes including fibrinolytic activation and dissolution, invasion by inflammatory cells, reepithelialization of the surface of the thrombus in contact with blood, and fibrosis (11). Serial imaging after acute DVT demonstrates residual thrombus in about $50 \%$ of patients at 6-12 mo, and this residual thrombus persists indefinitely (13-16). Given that recurrent DVT may be contralateral in $50 \%$ of patients, residual vein thrombus is thought to be in some way a manifestation of an underlying prothrombotic tendency.

A possible explanation of why residual thrombus is so prevalent involves plasma thrombin activatable fibrinolytic inhibitor (TAFI). Plasma TAFI is incorporated into developing thrombi and is activated by thrombin. Activated TAFI enzymatically cleaves the C-terminal lysine residues on partially degraded fibrin with the thrombus, removing binding sites for plasminogen activators such as tissue plasminogen activator and rendering the thrombus more resistant to fibrinolysis (17). Elevated plasma TAFI is associated with a prolonged in vitro clot lysis time for human plasma (18). In a model of tissue-factor-induced human plasma clotting, increased TAFI activation and prolonged clot lysis time were observed in FII G20210A heterozygotes compared with plasma controls (19). A similar pathogenesis has been proposed for the association between vein thrombosis and elevated factor XI (20) and factor V Leiden (21). Associations between TAFI levels and activity have been found in patients with DVT (22) and angina pectoris (23), although these findings are contradicted by other studies and further investigation is required.

We postulated that the action of TAFI on a newly formed thrombus will affect the uptake of ${ }^{99 \mathrm{~m}} \mathrm{Tc}$-modified recombinant tissue plasminogen activator $\left({ }^{99 \mathrm{~m}} \mathrm{Tc}-\mathrm{rt}-\mathrm{PA}\right)$ into the 
thrombus. ${ }^{99 \mathrm{~m}} \mathrm{Tc}-\mathrm{rt}-\mathrm{PA}$ imaging has previously been shown to have a high accuracy in the detection of acute proximal thrombosis and also distal DVT in both symptomatic and asymptomatic postarthroplasty patients $(24,25)$. Before radiolabeling with ${ }^{99 \mathrm{~m}} \mathrm{Tc}$, the plasminogen activation site of rt-PA is inhibited with minimal effect on the fibrin-binding site. In this way, uptake of this tracer into a thrombus is due to binding of fibrin within the thrombus. We propose that a newly formed thrombus will be ${ }^{99 \mathrm{~m}} \mathrm{Tc}-\mathrm{rt}-\mathrm{PA}$-avid. With aging of the thrombus, and TAFI-mediated cleavage of binding sites for rt-PA on fibrin within the thrombus, persistent thrombi will not take up this radiotracer and will become 99mTc-rt-PA-cold.

We therefore performed a study to investigate the uptake of ${ }^{99 \mathrm{~m}} \mathrm{Tc}-\mathrm{rt}-\mathrm{PA}$ in thrombi involving the deep veins of the legs over the first $4 \mathrm{wk}$ after diagnosis of acute DVT. Serial imaging of ${ }^{99 \mathrm{~m}} \mathrm{Tc}-\mathrm{rt}-\mathrm{PA}$ was correlated with serial compression ultrasound mapping of residual thrombi. The results of the study indeed suggest that imaging with ${ }^{99 \mathrm{~m}} \mathrm{Tc}-\mathrm{rt}-\mathrm{PA}$ does detect fresh thrombi and therefore may provide an easy imaging technique for differentiating new from old thrombi. This finding may have a practical clinical indication in the diagnosis of recurrent ipsilateral DVT.

\section{MATERIALS AND METHODS}

\section{Patients}

Patients referred to the Haematology Department at St. George Hospital, Sydney, over a 2-y period for investigation and management of acute symptomatic DVT were recruited to the study. Patients were eligible if they had had symptoms attributed to DVT for less than $7 \mathrm{~d}$, a positive compression ultrasound confirming DVT in the lower legs, and no past history of documented DVT in the ipsilateral leg. The protocol scanning requirements limited recruitment of patients to those presenting on Mondays to Thursdays inclusively. Of 400 eligible cases of DVT presenting for assessment during this period, 74 patients participated in the study. The Ethics Committee of South Eastern Sydney Area Health Service approved the study, and all participants gave written informed consent.

All patients presenting with DVT were treated in a standard fashion initially with enoxaparin $1.5 \mathrm{mg} / \mathrm{kg}$ once daily or $1 \mathrm{mg} / \mathrm{kg}$ twice daily by subcutaneous injection. Warfarin was commenced after 24-48 h of enoxaparin therapy, and enoxaparin was continued for a minimum of $5 \mathrm{~d}$, with at least 2 consecutive days of therapeutic INR (International Normalised Ratio). Most patients were treated as outpatients by one of the authors.

\section{Study Design}

Patients consenting to the study underwent ${ }^{99 \mathrm{~m}} \mathrm{Tc}$-rt-PA scanning within $24 \mathrm{~h}$. The day of their initial ${ }^{99 \mathrm{~m}} \mathrm{Tc}-\mathrm{rt}-\mathrm{PA}$ scan was called day 1 of the study. The radionuclide scan was reported in real time with reference to the diagnostic compression ultrasonography report and films. After initial ${ }^{99 \mathrm{~m}}$ Tc-rt-PA scanning, the patients were separated into 2 groups.

The first group ( $n=55$ ) consisted of patients positive for ${ }^{99 \mathrm{mTc}-}$ rt-PA uptake into the thrombus on day 1 . These patients underwent further ${ }^{99 \mathrm{~m}} \mathrm{Tc}-\mathrm{rt}-\mathrm{PA}$ scanning and compression ultrasonography on days 7 and 30. The results of compression ultrasonography were reported in real time in a standard fashion with reference to previous scans. ${ }^{99 \mathrm{~m}} \mathrm{Tc}-\mathrm{rt}-\mathrm{PA}$ scans were reported without knowledge of the previous scan results or ultrasound results.

The second group of patients $(n=19)$ had apparent confirmed DVT by ultrasonography but no ${ }^{99 m}$ Tc-rt-PA uptake into the thrombus and did not proceed with the study. Contrast venography was performed on 13 patients within $24 \mathrm{~h}$ of the ${ }^{99 \mathrm{~m}} \mathrm{Tc}-\mathrm{rt}-\mathrm{PA}$ scan. Of the other patients, 2 refused and 3 were unable to undergo contrast venography. On careful questioning of the remaining patient, the onset of symptoms attributable to DVT had occurred at least $4 \mathrm{wk}$ previously.

Venography excluded DVT in 11 of 13 patients, all with apparent tibioperoneal DVT. These patients ceased receiving anticoagulation treatment, and during 3 mo of follow-up none had experienced symptoms of vein thrombosis. Contrast venography found DVT in 2 of 13 patients, in the tibioperoneal trunk. In the first patient, a filling defect approximately $10 \mathrm{~cm}$ long was demonstrated in a solitary peroneal vein by ultrasonography and venography. In the second patient, a similarly sized filling defect was demonstrated in a peroneal vein extending into the popliteal vein by ultrasonography and venography. These 2 patients continued receiving anticoagulation treatment.

\section{Radionuclide Scanning}

Preparation of ${ }^{99 \mathrm{~m}} \mathrm{Tc}-\mathrm{rt}-\mathrm{PA}$, scanning, and scan interpretation were performed according to previously described methods $(24,25)$. Briefly, $1 \mathrm{GBq}$ of ${ }^{99 \mathrm{~m}} \mathrm{Tc}$ in $2.5 \mathrm{~mL}$ of normal saline was added to a lyophilized kit and incubated for $10 \mathrm{~min} .{ }^{99 \mathrm{~m}} \mathrm{Tc}-\mathrm{rt}-\mathrm{PA}(600 \mathrm{MBq})$ was administered by bolus injection into an antecubital fossa vein. Four hours later, imaging over the anterior thighs and posterior calves was performed using a Millennium $\gamma$-camera (GE Healthcare). Images were viewed on a video monitor using an interactive gray-scale threshold. The criteria for positive uptake were as follows: In the proximal vein, increased tracer accumulation in comparison with the opposite side such that the increase was equal to or greater than the adjacent bone marrow accumulation; in the calf veins, increased tracer accumulation in the deep veins in comparison with the opposite side; in the tibioperoneal trunk, increased tracer accumulation in comparison with the opposite side in a patient with coexisting calf or femoral thrombosis.

Representative scans of a normal study and of a study positive for ${ }^{99 m}$ Tc-rt-PA uptake are shown in Figures 1 and 2.

\section{Compression Ultrasound}

Ultrasonography was performed using a high-resolution 5-7.5 linear array transducer, with the course of the deep veins being evaluated for compressibility at various intervals from the groin to the ankle. Initial ultrasonography classified DVT as involving either the calf muscle vein, tibioperoneal trunk, or proximal veins (the trifurcation of the calf veins, popliteal vein, or more proximal veins.) All examinations on days 7 and 30 were performed at St. George Hospital and were directed toward the site of previous abnormality, with anatomic mapping of the extent of residual thrombus. Patients were classified by ultrasonography as having residual DVT (unchanged or persistent thrombus in previously involved segments) or having no residual thrombus (recanalized previously involved segments).

\section{Contrast Venography}

Contrast venography was performed according to standard methodology (26). Nonionic contrast material $(50-100 \mathrm{~mL})$ was injected into a dorsal foot vein of the limb suspected of DVT, after 
A

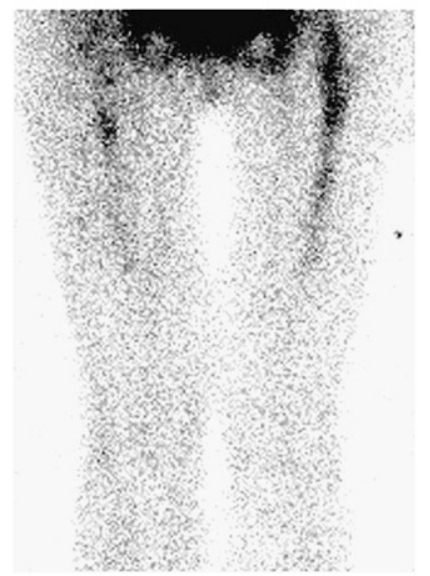

B

FIGURE 1. $99 \mathrm{~m}$ Tc-rt-PA scan of normal anterior thighs $(\mathrm{A})$ and posterior calves (B). Bone marrow accumulation in proximal femora is usually seen.

the placement of tourniquets at the ankle and the calf. Additional views were taken as needed.

\section{RESULTS}

\section{Study Population}

The study population comprised 74 patients referred to the Haematology Department with acute symptomatic DVT (41 women and 33 men; mean age, $56 \mathrm{y}$ ). The location of the thrombus on day 1 ultrasonography was the calf muscle vein in 9 patients, tibioperoneal trunk in 30 patients, and proximal vein in 35 patients. The results of the imaging studies for the 74 patients are detailed in Figure 3. Fiftyfive patients with DVT found on ultrasonography (31 proximal, 15 tibioperoneal trunk, 9 calf muscle) had scans positive for ${ }^{99 m}$ Tc-rt-PA uptake on day 1 and proceeded with the study protocol. All 55 patients underwent day 7 imaging studies, and 44 patients ( 8 calf muscle, 12 tibiopero-
A

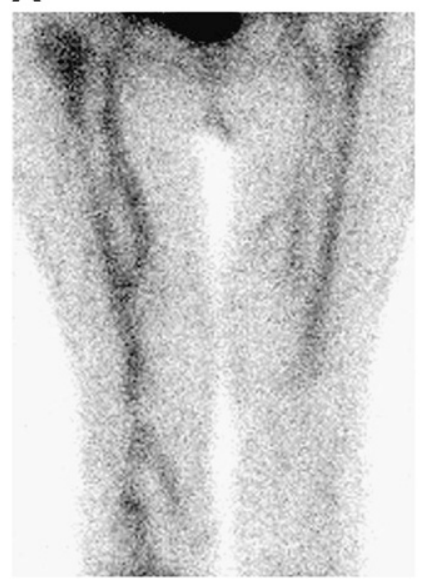

B

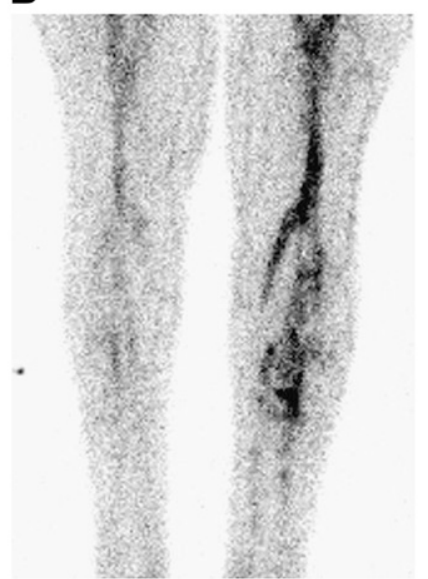

FIGURE 2. ${ }^{99 m}$ Tc-rt-PA scan of anterior thighs $(A)$ and posterior calves $(B)$ in a patient with extensive DVT involving calf, tibioperoneal, and proximal veins.

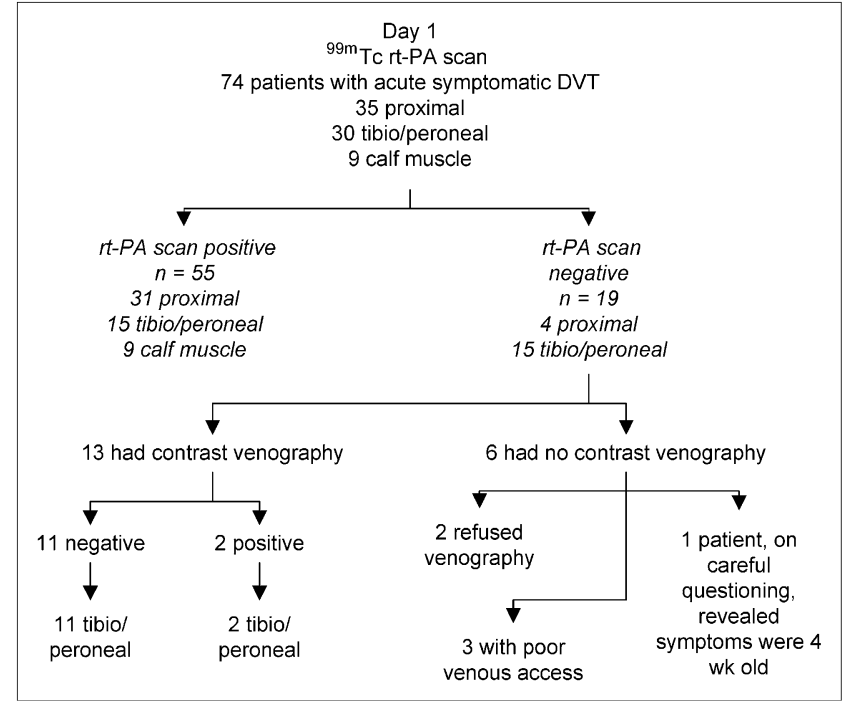

FIGURE 3. Results of imaging on day 1.

neal, 24 proximal DVT) completed the day 30 imaging studies (Figs. 4 and 5).

Three patients deteriorated clinically between days 7 and 30 and were unable to complete the protocol, and a further 8 patients either refused or were unable to undergo imaging studies.

\section{Sequential Ultrasound and Radionuclide Imaging}

Serial ultrasound showed persistent thrombi in 46 (84\%) of 55 patients on day 7 and in $29(66 \%)$ of 44 of patients on day 30 . Of the persisting thrombi on day 7, $33(72 \%)$ of 46 showed ${ }^{99 \mathrm{~m}} \mathrm{Tc}-\mathrm{rt}-\mathrm{PA}$ uptake. Of the persisting thrombi on day 30, $0(0 \%)$ of 29 showed ${ }^{99 \mathrm{~m}} \mathrm{Tc}-\mathrm{rt}-\mathrm{PA}$ uptake.

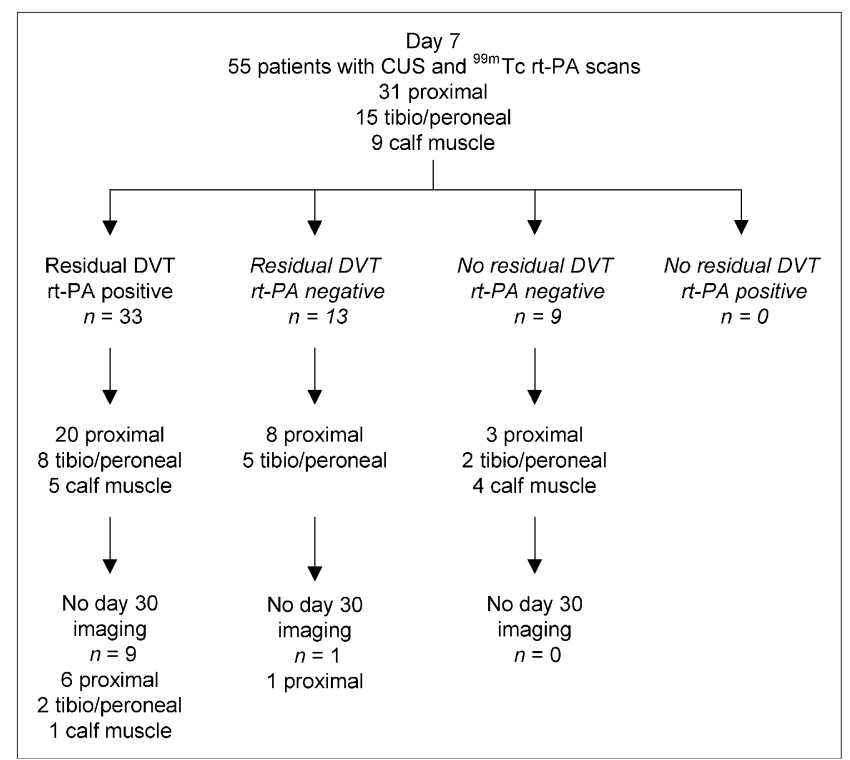

FIGURE 4. Results of imaging on day 7. CUS = carotid ultrasound examination.

99MTc-RT-PA IN DVT IMAGING • Brighton et al. 


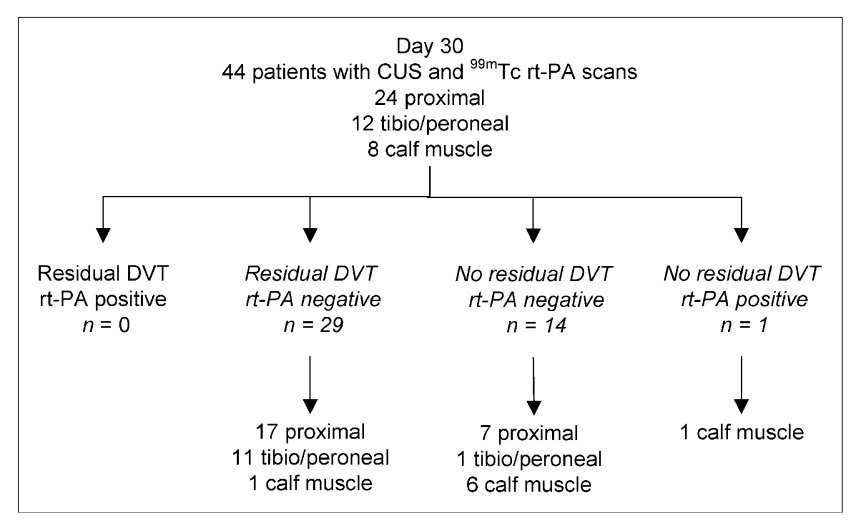

FIGURE 5. Results of imaging on day 30. CUS = carotid ultrasound examination.

Serial ultrasound showed that 17 patients recanalized their DVT during the study. In all but 1 patient, serial ${ }^{99 \mathrm{~m} T c-r t-}$ PA scanning showed no uptake at the site of the original thrombus. In this 1 patient, the DVT was localized to the calf veins on days 1 and 7, with normal ultrasound findings on day 30. ${ }^{99 m}$ Tc-rt-PA was positive on days 1,7 , and 30 . Contrast venography was not performed, and the patient remained asymptomatic on anticoagulant therapy. Representative scans of sequential imaging performed on patients with proximal vein thrombosis and a calf vein thrombosis are shown in Figures 6 and 7.

\section{DISCUSSION}

Radionuclide scanning with ${ }^{99 \mathrm{~m} T c-r t-P A}$ is a novel technique in which the plasminogen activation site of rt-PA is inactivated, leaving the fibrin-binding site unaltered and able to bind to fibrin in a thrombus. Previous studies by our group have demonstrated the high sensitivity and specificity of ${ }^{99 m}$ Tc-rt-PA scanning for symptomatic and asymptomatic DVT. In this study, ${ }^{99 \mathrm{~m}} \mathrm{Tc}-\mathrm{rt}-\mathrm{PA}$ uptake into an acute thrombus declines with time so that at $30 \mathrm{~d}$, there is no appreciable uptake into the thrombus.

Many patients with a history of acute DVT present again after their treatment with new symptoms that may be caused by a further DVT. Discrimination of recurrent ipsilateral DVT from postphlebitic symptoms is essential, because recurrent DVT merits further anticoagulation therapy that may need to be life-long. An objective determination of the age of deep vein thrombi is not readily achieved with the currently available techniques. Ultrasound and venography techniques have been reported to be able to differentiate between fresh and organized thrombi, but these methods are difficult to validate in the clinical setting. A recent study in a porcine model of inferior vena cava thrombosis found that reduced thrombus elasticity, as determined by ultrasonography of gelatin-embedded thrombi, correlated with histologic findings of increased fibroblasts and collagen content during the first $2 \mathrm{wk}$ after thrombus generation (27).

Confirmation of recurrent ipsilateral DVT by ultrasonography requires a new segment of vein incompressibility that was not present on the initial ultrasound. Prandoni et al. $(13,14)$ have proposed that serial measurement of proximal vein diameters during compression can be accurate in diagnosing a new thrombosis and provide adequate information on which to base treatment decisions. To be useful clinically, both approaches require detailed mapping of residual DVT and serial ultrasonography, which are not routinely available in many cases. The results of the current study suggest that uptake of ${ }^{99 \mathrm{~m}} \mathrm{Tc}$-rt-PA into vein thrombi indicates the thrombus is new or young and at least $30 \mathrm{~d}$ old.

An extension of the need to differentiate between fresh and organized thrombi would be the potential to select patients for thrombolysis. Efficient lysis of thrombi without damaging venous endothelium or vein valves is limited to a thrombus age of $7-10 \mathrm{~d}(28,29)$.

The pathophysiology underlying the diminished uptake of ${ }^{99 m}$ Tc-rt-PA in organized thrombi is unknown at this time. Although it is likely that TAFI cleavage of the rt-PA binding sites on fibrin is involved in the aging of thrombi, the exact factors involved are not well understood. In broad terms, it appears that while the fibrin is still active, there are adequate binding sites for ${ }^{99 \mathrm{~m}} \mathrm{Tc}-\mathrm{rt}-\mathrm{PA}$. As the thrombus ages, the fibrin is not active and there is no binding of ${ }^{99 m}$ Tc-rt-PA.

Of considerable interest is the observation that several patients with positive ultrasound findings did not have

FIGURE 6. Sequential radionuclide images obtained on days $1(\mathrm{~A}), 7(\mathrm{~B})$, and 30 (C) in patient with left proximal vein thrombosis. Day 1 shows marked accumulation of $99 \mathrm{~m}$ Tc-rt-PA in left proximal vein, with urinary contamination over medial left calf being noted. Day 7 scan shows appreciably less uptake in thrombus. Day 30 shows no significant uptake. Ultrasound was positive for proximal vein thrombosis on days 1,7 , and 30 .

A

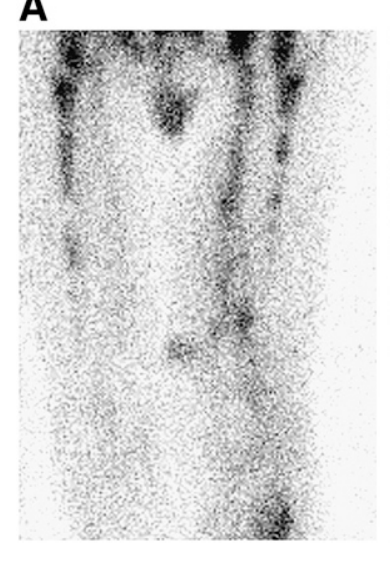

B

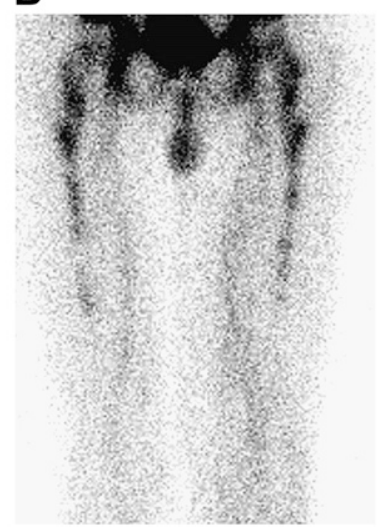

C

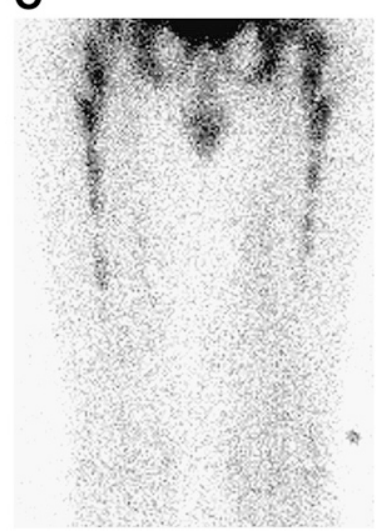


A

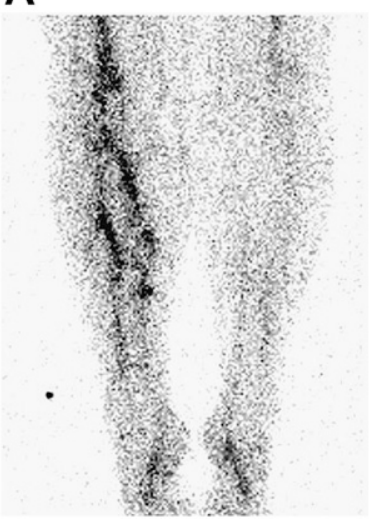

B

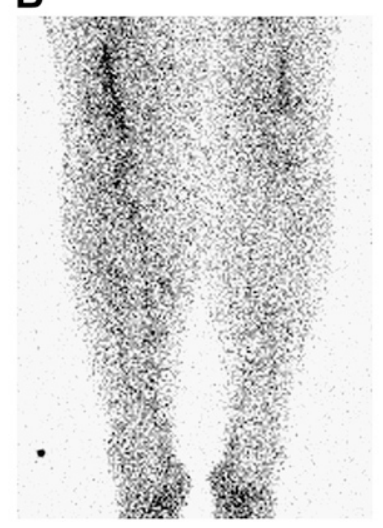

C

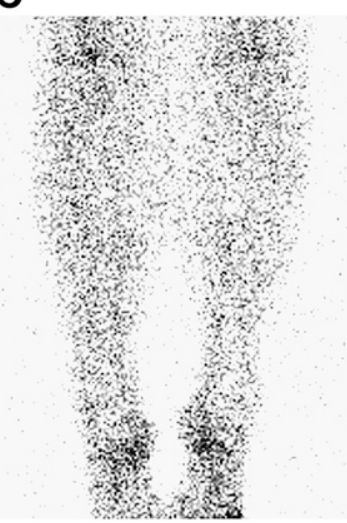

FIGURE 7. Sequential radionuclide images obtained on days $1(\mathrm{~A}), 7(\mathrm{~B})$, and 30 (C) in patient with left calf muscle vein thrombosis extending to tibioperoneal trunk. Day 1 shows marked accumulation of $99 \mathrm{~m}$ Tc-rt-PA in left leg. Day 7 scan shows appreciably less uptake in thrombus. Day 30 shows no significant uptake. Ultrasound was positive for thrombosis on days 1,7 , and 30 . objective evidence of DVT on contrast venography. Of the 74 patients initially recruited to the study, 19 had scans negative for ${ }^{99 \mathrm{~m}} \mathrm{Tc}-\mathrm{rt}-\mathrm{PA}$ uptake, with 13 of the 19 proceeding to contrast venography to confirm the presence of DVT. All these patients were considered to have isolated calf vein thrombosis on carotid ultrasound examination. In those patients with diagnostic contrast venography, 11 of 13 patients had no evidence of a thrombus in the calf. There are several reasons for this finding. First, ultrasound has a specificity of approximately $60 \%-70 \%$ for isolated calf vein thrombosis, and false-positive cases are expected (30-32). Second, most of these patients had the ultrasound performed at outside radiology practices. Ultrasound directed at the calf is technically difficult and may not have been optimally performed and interpreted. Radionuclide scanning with ${ }^{99 \mathrm{~m}} \mathrm{Tc}-\mathrm{rt}-\mathrm{PA}$ in patients with isolated calf vein thrombosis has a sensitivity of $86 \%$ and a specificity of $93 \%$, and the 2 false-negative cases observed in this study were in keeping with this reported accuracy.

Our study had some limitations. First, it was a small study designed only to explore the uptake of ${ }^{99 \mathrm{~m} T \mathrm{Tc}-\mathrm{rt}-\mathrm{PA} \text { into }}$ thrombi during a 30-d period, and the work needs to be confirmed by other investigators. Second, no commercial preparation of this tracer is available, and with the widespread availability of ultrasound, the clinical application of this technique is likely to be limited. Nevertheless, the possibility of discriminating new from old thrombi would be clinically useful, and radionuclide scanning with ${ }^{99 \mathrm{~m}} \mathrm{Tc}-$ rt-PA may be helpful.

\section{CONCLUSION}

Many patients with a past history of DVT present again with further complaints of pain and swelling in the limb that had the DVT. Ultrasound can often detect a thrombus in the affected limb, but differentiation between a recent thrombus formation and persisting thrombus is difficult. Scanning with ${ }^{99 \mathrm{~m}} \mathrm{Tc}-\mathrm{rt}-\mathrm{PA}$ may be able to assist in this clinical problem because the current study demonstrated that uptake of ${ }^{99 \mathrm{~m} T c-r t-P A}$ into DVT is absent $30 \mathrm{~d}$ after diagnosis. This finding suggests that this imaging technique can distinguish fresh from old thrombus.

\section{ACKNOWLEDGMENTS}

The authors are indebted to Dr. Richard Quinn for his assistance with reporting the ${ }^{99 \mathrm{~m}} \mathrm{Tc}-\mathrm{rt}-\mathrm{PA}$ findings and to the Radiology Department of St. George Hospital for their expert assistance in performing and interpreting the compression ultrasound and contrast venography studies.

\section{REFERENCES}

1. Andersson FA Jr, Wheeler HB, Goldberg RJ, et al. A population-based perspective of the hospital incidence and case-fatality rates of deep vein thrombosis and pulmonary embolism: The Worchester DVT Study. Arch Intern Med. 1991;151:933-938.

2. Silverstein MD, Heit JA, Mohr DN, Petterson TM, O'Fallon WM, Melton LJ III. Trends in the incidence of deep vein thrombosis and pulmonary embolism: a 25year population-based study. Arch Intern Med. 1998;158:585-593.

3. Ho WK, Hankey GJ, Lee CH, Eikelboom JW. Venous thromboembolism: diagnosis and management of deep vein thrombosis. Med J Aust. 2005;182:476481.

4. Kearon C, Salzman EW, Hirsh J. Epidemiology, pathogenesis, and natural history of venous thromboembolism. In: Colman RW, Hirsh J, Marder VJ, Clowes AW, George JN. Hemostasis and Thrombosis: Basic Principles and Clinical Practice. 4th ed. Philadelphia, PA: J.B. Lippincott Williams and Wilkins; 2001:1153-1177.

5. Baglin T, Luddington R, Brown K, Baglin C. Incidence of recurrent venous thromboembolism in relation to clinical and thrombophilic risk factors: prospective cohort study. Lancet. 2003;362:523-526.

6. Hansson P-O, Sorbo J, Eriksson H. Recurrent venous thromboembolism after deep venous thrombosis. Arch Intern Med. 2000;160:769-774.

7. Prandoni P, Lensing AWA, Cogo A, et al. The long-term clinical course of acute deep vein thrombosis. Ann Intern Med. 1996;125:1-7.

8. Mohr DN, Silverstein MD, Heit JA, Petterson TM, O'Fallon WM, Melton LJ III. The venous stasis syndrome after deep vein thrombosis or pulmonary embolism: a population-based study. Mayo Clin Proc. 2000;75:1249-1256.

9. Kahn SR, Kearon C, Julian JA, et al. Predictors of the post-thrombotic syndrome during long-term treatment for proximal deep vein thrombosis. J Thromb Haemost. 2005;3:718-723.

10. Kearon C, Ginsberg JS, Hirsh J. The role of venous ultrasonography in the diagnosis of suspected deep venous thrombosis and pulmonary embolism. Ann Intern Med. 1998;129:1044-1049.

11. Heijboer H, Jongbloets LM, Buller HR, Lensing AW, Ten Cate JW. Clinical utility of real-time compression ultrasonography for diagnostic management of patients with recurrent venous thrombosis. Acta Radiol. 1992;4:297-300.

12. Freiman DG. The structure of thrombi. In: Coleman RW, Hirsch J, Marder VJ, Salzman EW. Hemostasis and Thrombosis: Basic Principles and Clinical Practice. 2nd ed. Philadelphia, PA: Lippincott Williams and Wilkins; 1987:1123-1135.

13. Prandoni $\mathrm{P}, \mathrm{Cogo} \mathrm{A}$, Bernardi E, et al. A simple ultrasound approach for the detection of recurrent proximal-vein thrombosis. Circulation. 1993;88:1730-1735.

14. Prandoni P, Lensing AW, Prins MH, et al. Residual venous thrombosis as a predictive factor for recurrent venous thromboembolism. Ann Intern Med. 2002;137:955-960. 
15. Piovella F, Crippa L, Barone $\mathrm{M}$, et al. Normalization rates of compression ultrasonography in patients with a first episode of deep vein thrombosis of the lower limbs: association with recurrence and new thrombosis. Haematologica. 2002;87:515-522.

16. Siragusa S, Mariani G. Residual vein thrombosis assessment establishes the optimal duration of oral anticoagulants in patients with idiopathic or provoked deep vein thrombosis: a randomised, controlled trial [abstract]. Blood. 2003;102:55a.

17. Mutch NJ, Moore NR, Wang E, Booth NA. Thrombus lysis by uPA, scuPA and tPA is regulated by plasma TAFI. $J$ Thromb Haemost 2003;1:2000-2007.

18. Mosnier LO, von dem Borne PA, Meijers JC, Bouma BN. Plasma TAFI levels influence clot lysis time in healthy individuals in the presence of an intact intrinsic pathway of coagulation. Thromb Haemost. 1998;80:829-835.

19. Colucci M, Binetti BM, Tripodi A, Chantarangkul V, Semararo N. Hyperprothrombinaemia associated with prothrombin G20210A mutation inhibits plasma fibrinolysis through a TAFI-mediated mechanism. Blood. 2004;103: 2157-2161.

20. Meijers JC, Tekelenburg WL, Bouma BN, Bertina RM, Rosendaal FR. High levels of coagulation factor $\mathrm{XI}$ as a risk factor for venous thrombosis. $N$ Engl J Med. 2000;342:696-701.

21. Bajzar L, Kalafatis M, Simioni P, Tracy PB. An antifibrinolytic mechanism describing the prothrombotic effect associated with factor V Leiden. J Biol Chem. 1996;271:22949-22952.

22. Van Tilburg NH, Rosendaal FR, Bertina RM. Thrombin activatable fibrinolysis inhibitor and the risk for deep vein thrombosis. Blood. 2000;95:2855-2859.
23. Morange PE, Juhan-Vague I, Scarabin PY, et al. Association between TAFI antigen and Ala147Thr polymorphism of the TAFI gene and the angina pectoris incidence. Thromb Haemost. 2003;89:554-560.

24. Butler SP, Boyd SJ, Parkes SL, Quinn RJ. Technetium-99m modified recombinant tissue plasminogen activator to detect venous thrombosis. J Nucl Med. 1996;37:744-748.

25. Butler SP, Rahman T, Boyd SJ, Parkes SL, Quinn RJ. Accurate detection of asymptomatic post operative deep venous thrombosis using technetium-99m labelled modified recombinant tissue plasminogen activator. J Nucl Med. 1997;38:219-223.

26. Rabinov K, Paulin S. Roentgen diagnosis of venous thrombosis in the leg. Arch Surg. 1972;104:134-144.

27. Geier B, Barbera L, Muth-Werthman D, et al. Ultrasound elasticity for the age determination of venous thrombi: evaluation in an animal model of venous thrombosis. Thromb Haemost. 2005;93:368-374.

28. Stiegler H, Arbogast H, Nees S, Halder A, Grau A, Riess H. Thrombectomy, lysis, or heparin treatment: concurrent therapies of deep vein thrombosis: therapy and experimental studies. Semin Thromb Hemost. 1989;15:250-258.

29. Marder VJ, Sherry S. Thrombolytic therapy. N Engl J Med. 1988;318:1512-1520.

30. Hull RD, Carter CI, Jay RM, et al. The diagnosis of acute recurrent DVT: a diagnostic challenge. Circulation. 1983;67:901-906.

31. Hirsh J, Lee AYY. How we diagnose and treat deep vein thrombosis. Blood. 2002;99:3102-3110.

32. Prandoni P, Lensing AWA, Prins MH, et al. The diagnostic value of compression ultrasonography in patients with suspected recurrent deep vein thrombosis. Thromb Haemost. 2002;88:402-406. 